\title{
CYCLE ÉVOLUTIF DE SYNHIMANTUS (DESPORTESIUS) SPINULATUS (NEMATODA ACUARIIDAE)
}

\author{
Par A.-G. CHABAUD
}

Sous la membrane du gésier d'un pique-bœuf Bubulcus ibis (L. 1758), tué à Had Kourt, dans le Rharb (Maroc), nous avons récolté une vingtaine d'exemplaires d'un nématode que nous avions récemment décrit, en collaboration avec Y. Campana, sous le nom de Synhimantus (Desportesius) equispiculatus Wu et Liu 1943, var. spinulatus.

Dans la première partie de ce mémoire, nous reviendrons rapidement sur la morphologie de l'adulte et exposerons les raisons qui nous conduisent à élever la variété au rang d'espèce.

Dans une seconde partie, nous décrirons le cycle évolutif et la morphologie des différents stades larvaires.

\section{I. - MORPHOLOGIE DE L'ADULTE}

Matériel. - Nous disposons, d'une part, de sept mâles et de quatre femelles récoltés chez un Bubulcus spontanément infesté et, d'autre part, de sept mâles, sept femelles matures et une femelle juv. obtenus à l'autopsie de l'oiseau infesté au laboratoire.

Morphologie. - Les spécimens marocains ont la même anatomie que ceux qui ont été récoltés en France, mais ils en diffèrent par leur grande taille (longueur maximum du mâle : $12,5 \mathrm{~mm}$., au lieu de $8,9 \mathrm{~mm}$., et femelle : $14 \mathrm{~mm}$., au lieu de $9,9 \mathrm{~mm}$ ).

Les modifications qu'amène ce facteur dans les différentes mensurations sont consignées dans un tableau. Ces chiffres confirment les conclusions que nous avions énoncées (cf. p. 89) :

La longueur relative des spicules est un élément insuffisant pour la diagnose.

Ann. de Parasitologie, T. XXV, $\mathrm{x}^{\circ} 3 .-1950$, p. 150-166. 


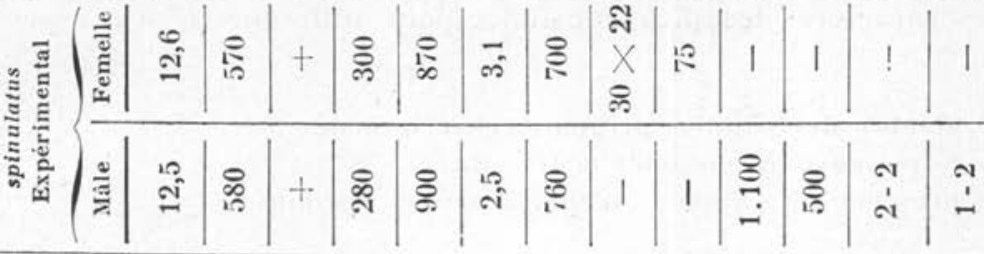

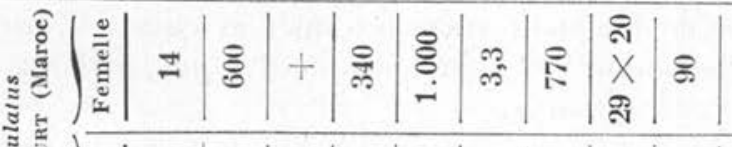

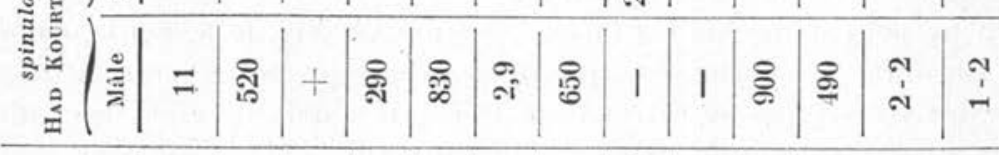

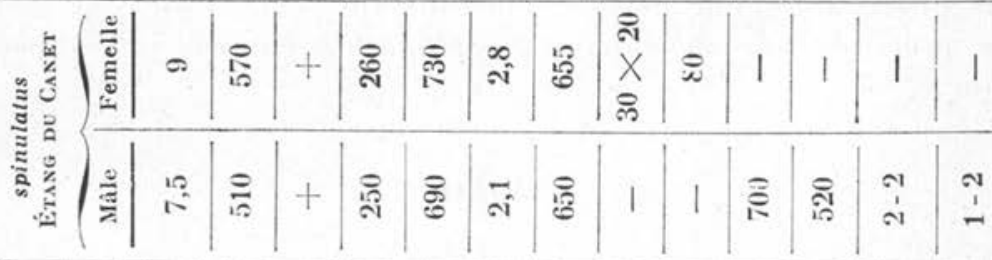

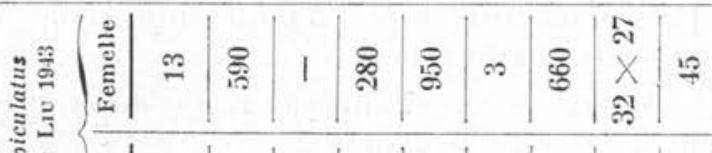

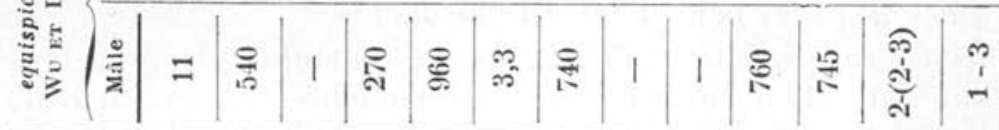

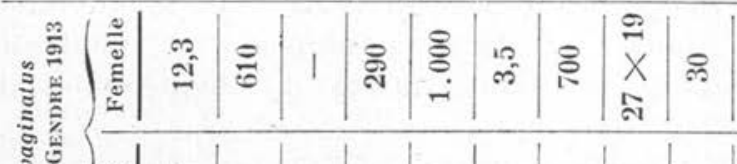

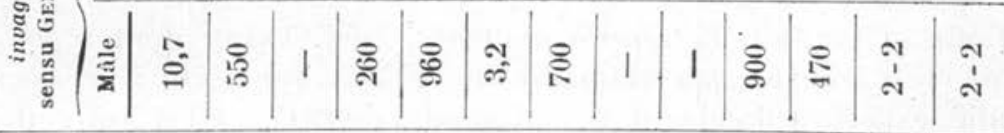

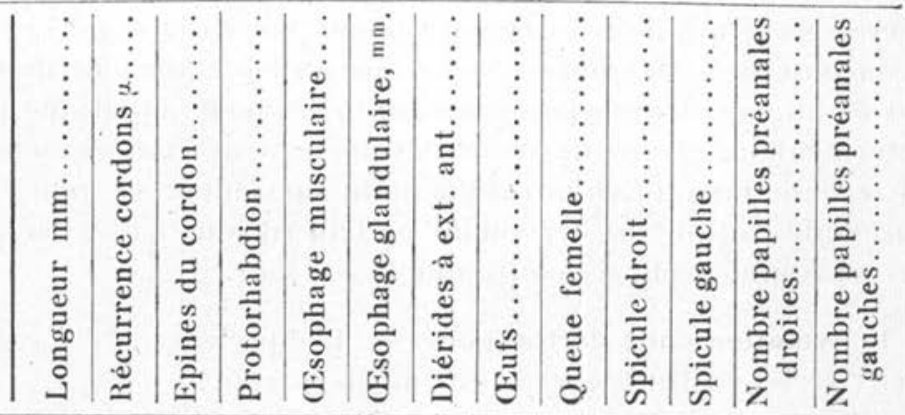


Les caractères les plus constants pour différencier les espèces sont :

Le nombre des papilles préanales chez le mâle.

La forme de la queue chez la femelle.

La présence ou l'absence d'épines sur les cordons.

La forte différence de longueur entre les spicules chez les spécimens de grande taille éloigne ces nématodes de $S$. equispiculatus et les rapprochent de $S$. invaginatus.

On ne peut donc pas les laisser comme variété de $S$. equispiculatus. En outre, les caractères que nous avions proposés pour la diagnose des trois types se retrouvent de façon constante chez des individus provenant d'une espèce d'oiseau différente. Nous croyons donc préférable de considérer $S$. spinulatus comme une espèce distincte.

\section{II. - BIOLOGIE}

Les helminthes les plus proches du genre Synhimantus dont le cycle évolutif est connu sont les suivants :

Dispharynx spiralis (Molin 1858), étudié par E. Cram en 1931, qui a des isopodes pour hôtes intermédiaires.

Cheilospirura spinosa (Cram 1927) et C. hamulosa (Diesing 1851), qui ont fait l'objet de recherches assez nombreuses, en particulier Cram 1931, Cuvillier 1933, Alicata 1938, Refuerzo 1940. L'hòte intermédiaire est peu spécifique: crustacés amphipodes et nombreux insectes dans les familles les plus variées d'orthoptères et de coléoptères.

Enfin et surtout, Echinuria uncinata (Rud. 1819), dont le cycle avait été découvert par Hamann en 1893 et qui a été récemment étudié expérimentalement par Romanova (1947). C'est cette dernière espèce qui nous intéresse surtout, car sa biologie se rapproche beaucoup de $S$. spinulatus. Seules, parmi les Acuariidæ dont le cycle est établi, ces deux espèces ont un arthropode aquatique pour hòte intermédiaire et une durée de développement extrêmement courte : la seconde mue d'Echinuria uncinata survient le $12^{\circ}$ jour. La maturation de l'adulte est cependant nettement plus lente, ear les femelles ne commencent à pondre que le $51^{\circ}$ jour.

I. Evolution chez l'arthropode. - L'expérience a été conduite en deux séries distinctes, espacées de six jours. 
A) Un premier bocal reçoit quatre femelles matures et le contenu intestinal du Bubulcus. Vingt-quatre heures après, les arthropodes suivants sont introduits dans le récipient : environ 200 Ostracodes des deux espèces Cyprinotus salinus (Brady 1868) et Pionocypris vidua vidua (O. F. Müller 1776), 50 Copépodes sp., 4 larves de Theobaldia longeareolata (Macquart 1838), 3 larves d'Ephémère sp. et une larve de Chironome sp.

B) Un second bocal, infesté seulement par trois femelles matures, est abandonné pendant sept jours. A ce moment seulement sont introduits dans le milieu environ 200 Ostracodes des mêmes espè. ces que précédemment. Les élevages sont conservés à une température de $25^{\circ}$.

Les $T$. longevareolata examinés les $6^{\circ}$ et $7^{\circ}$ jours présentent dans l'intestin de très nombreux œufs non embryonnés qui sont restés intacts et des coques d'œufs ouverts aux deux pôles (Fig. 1 C) ; une seule larve a été retrouvée au moment précis où elle sortait de l'œuf.

Les Ephémères étudiés les $5^{\circ}$ et $8^{\circ}$ jours contiennent également de nombreuses coques d'œufs, mais pas de formes larvaires.

La larve de Chironome sacrifiée le $7^{\circ}$ jour est parasitée par une jarve d'un nématode non déterminé, très différente de l'espèce qui nous occupe ici.

Les Cyclops étudiés le $5^{\circ}$ jour montrent un petit nombre de larves jeunes du premier stade dans l'intestin et, dans un cas seulement, une larve parvenue dans la cavité générale. Les $7^{\circ}, 8^{\circ}$ et $11^{\circ}$ jours, ils restent constamment négatifs.

Les deux espèces d'Ostracodes du premier bocal sont parasitées de façon constante et souvent très abondante. Aux environs du $10^{\circ}$ jour, il se produit une mortalité importante, qui est causée par l'excès du parasitisme. En effet, les cadavres renferment de 8 à 14 larves de la fin du deuxième stade, alors que les Cypris survivants ont toujours moins de parasites. Le nombre maximum de larves compatible avec la vie de l'hôte paraît être d'environ huit chez Cyprinotus salinus adulte et de six chez Pionocypris vidua vidua. (La forme adulte de cette dernière espèce a une longueur qui ne dépasse guère $0,6 \mathrm{~mm}$., alors que $C$. salinus atteint $1 \mathrm{~mm}$.).

Dans le second bocal, le taux du parasitisme est plus faible : 50 p. 100 des Ostracodes sont indemnes et les exemplaires positifs n'hébergent jamais plus de trois larves.

La conservation des œufs dans l'eau explique que les Cypris renferment des larves à des stades parfois très différents, car ils ont eu la faculté de se réinfester pendant plusieurs jours. Nous n'avons done tenu compte pour apprécier la durée du développe- 
ment que des larves les plus évoluées. En outre, à partir du deuxième stade, les plus grosses larves proviennent toujours d'un Ostracode de grande taille (femelle de $C$. salinus) peu parasité. Le pluriparasitisme amène une réduction de la taille des larves d'environ 25 p. 100.

En tenant compte de ces facteurs, nous obtenons les résultats suivants : la larve, tout de suite après l'éclosion, passe dans la cavité générale. Rapidement, elle s'immobilise et prend un aspect un peu plus trapu, qui correspond à un début d'organisation interne. La taille n'augmente pas jusqu'à la première mue qui a lieu le $5^{\circ}$ jour.

Le $6^{\circ}$ jour, les larves ont subi une transformation considérable. Elles ont plus que doublé tant en longueur qu'en largeur. La description morphologique faite plus bas montrera les analogies frappantes qui existent entre ces larves et les stades en « saucisse » de filaires extraites des muscles thoraciques de moustiques. Le développement à cette période est extrêmement rapide, puisque, en 48 heures, les larves doublent leur taille. Les $8^{\circ}$ et $9^{\circ}$ jours, la croissance est très ralentie, les larves préparent la deuxième mue, qui a lieu le $9^{\circ}$ ou le $10^{\circ}$ jour.

Cette seconde mue va donner lieu, à nouveau, à une croissance brutale et brève, la larve du troisième stade atteignant d'emblée une longueur double de la précédente. La croissance ainsi réalisée est effectuée dans un temps si court que, malgré des examens très nombreux, nous n'avons pas pu trouver une seule larve intermédiaire entre les spécimens d'environ $700 \mu$ de la fin du deuxième stade et les larves du troisième stade qui mesurent au moins $1.250 \mu$. A parlir du $10^{\circ}$ jour, la croissance est extrêmement réduite et la larve parait être parfaitement mature dès le $12^{\circ}$ ou $13^{\circ}$ jour.

II. Réencapsulement. - Trois espèces de petits vertébrés ont été expérimentées dans ce sens. Quatorze jours après le début de l'expérience, ils ingèrent chacun soit deux ostracodes, soit quelques larves mises en liberté après écrasement de l'hôte.

a) Deux jeunes têtards de Discoglossus pictus Ott. 1837. Le premier examiné après 48 heures présente dans la cavité générale une iarve libre bien mobile, longue de $1.300 \mu$. Le second, sacrifié 16 jours après son infestation, contient plusieurs larves vivantes encapsulées dans le tissu graisseux du péritoine.

b) Deux Gambusia sp. L'un, infesté par des larves mises en liberté, est sacrifié 5 heures après. On retrouve deux larves vivantes dans la lumière du tube digestif, mais aucune n'a traversé la paroi intestinale. Le second Gambusia, infesté à plusieurs reprises par des 
larves libres et par des Cypris vivants, est sacrifié 16 jours après et reste négatif.

c) Un Cyprinidé juv., long de $2 \mathrm{~cm}$., proche de Barbus, sacrifié le $4^{\circ}$ jour, permet de recueillir trois larves encapsulées à la surface de l'intestin dans un tissu déjà résistant.

Quel que soit l'hôte, les capsules sont subsphériques (ou parfois comprimées sur deux faces) et ont un diamètre de 300-450 $\mu$.

III. Evolution chez l'hôte définitif. - Deux Bubulcus ibis adultes sont capturés à Tit Mellil, le $1^{\text {er }}$ octobre ; l'un sacrifié comme témoin est parasité par de nombreux Microtetrameres spiralis (Seurat 1915) dans le ventricule succenturié et par des Gamasides sp. dans les fosses nasales, mais n'a aucun Acuariidx sous la membrane du gésier.

Le second est infesté artificiellement à deux reprises : $1^{\circ}$ le $1^{\text {er }}$ octobre, par 10 Ostracodes du premier élevage (Cypris infestés depuis $2 \theta$ jours) ; $2^{\circ}$ le 9 octobre, par environ 30 Ostracodes du deuxième élevage (Cypris infestés depuis 22 jours).

L'oiseau est sacrifié le 14 octobre et l'autopsie permet de découvrir :

a) dans l'intestin : Nephrostomum ramosum (P. Sonsino 1895) (déterm. R. Ph. Dollfus);

b) dans le ventricule succenturié : Microtetrameres spiralis (Seurat 1915);

c) sous la couche cornée et dans la muqueuse du gésier : des Sỵnhimantus à deux stades très différents. Les premiers, longs de 2 à $4 \mathrm{~mm}$., sont des larves du $4^{\circ}$ stade ingérées par l'hòte depuis 6 jours. Les seconds, longs de 8 à $14 \mathrm{~mm}$., sont des adultes qui correspondent à la première infestation datant de 15 jours. Les mâles ne se distinguent en rien des exemplaires récoltés au départ chez le Bubulcus spontanément infesté. Sur les huit femelles, une seule est nettement juvénile, longue de $8 \mathrm{~mm}$., avec des œufs non embryonnés. Les autres sont matures et ont des œufs parfaitement cmbryonnés dans l'ovéjecteur. Un caractère cependant indique la jeunesse de ces exemplaires : la cupule cuticulaire qui engaine la queue est encore en voie de formation et l'extrémité postérieure de la pointe caudale reste libre (1).

(1) Une infestation préalable de l'oiseau ayant servi à l'expérience est peu probable étant donné les élémients suivants témoin négatif; rareté de l'espèce S. spinulatus; absence d'oufs de Synhimantus dans les selles; présence à l'autopsie de deux stades très différents correspondant à deux infestations expérimentales espacées de neuf jours. 
N'ayant pu étudier que deux stades chez l'hòte définitif, nous ne pouvons pas décrire de façon précise l'allure du développement. Ces résultats suffisent cependant à prouver l'extraordinaire rapidité de la croissance. La troisième mue s'effectue avant le $6^{\circ}$ jour et la quatrième mue nettement avant le $15^{\circ}$ jour, puisque à cette date les femelles sont déjà matures.

\section{III. - MORPHOLOGIE DES STADES LARVAIRES}

Premier stade larvaire. - Les larves récoltées chez les Cypris durant les quatre premiers jours se présentent sous deux aspects

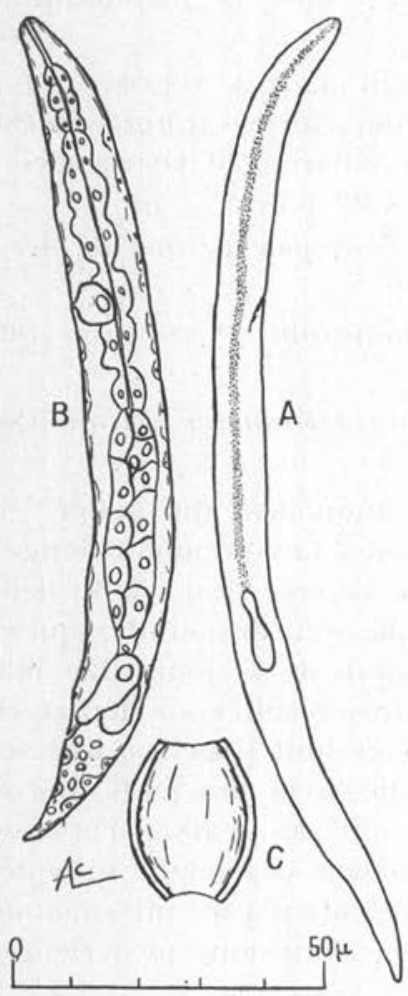

Fig. 1. - S. spinulatus. Premier stade larvaire. - A) Larve très jeune; vue latérale. - B) Larve au $4^{e}$ jour ; vue latérale. - C) (Euf ouvert aux deux pôles dans l'intestin d'un arthropode.

un peu différents, avec des stades intermédiaires entre les deux extrêmes.

1. - Une larve relativement longue $(155 \mu)$ et mince $(8 \mu)$, légèrement, mobile, à structure peu différenciée (Fig. $1 \mathrm{~A}$ ). 
2. - Une larve plus courte $(130 \mu)$ et plus large $(12 \mu)$, presque immobile, avec un début d'organisation cellulaire (Fig. $1 \mathrm{~B}$ ).

La forme longue correspond à la larve récemment éclose, recueillie peu après son passage à travers l'intestin. On ne distingue qu'une ligne noirâtre, allant de la bouche à une grande vésicule située à $100 \mu$ de l'apex. Cette vésicule représente probablement l'ébauche de l'ampoule rectale. Le pore excréteur apparait à $45 \mu$ de l'extrémité antérieure.

La forme courte correspond à la fin du premier stade larvaire. L'appareil digestif est maintenant mieux individualisé. Il comprend successivement un tube pharyngien d'environ $10 \mu$, une portion osophagienne longue de $60 \mu$, marquée par de grandes cellules espacées entourant la lumière osophagienne ; une portion intestinale fusiforme longue de $40 \mu$, formée par un massif cellulaire plus dense ; enfin, plus en arrière, l'ébauche rectale est représentée par deux très grosses cellules ovalaires. La cellule excrétrice est située à $45 \mu$ de l'apex.

Deuxième stade larvaire. - Les larves extraites des Cypris sont presque immobiles. Elles sont très fragiles et éclatent rapidement dans l'eau ordinaire. Dans l'eau physiologique, au contraire, elles peuvent rester vivantes pendant presque une heure.

Le deuxième stade larvaire, bien qu'il soit très éphémère (du $5^{\circ}$ au $10^{\circ}$ jour), donne lieu à un très rapide accroissement de taille. Nous devons donc décrire séparément la larve jeune et la larve àgée.

La larve qui a mué récemment (Fig. $2 \mathrm{~A}, \mathrm{~B}$ ) mesure environ $300 \mu$ en longueur et $30 \mu$ en largeur. Sa forme trapue et la dilatation de la région postérieure produite par le grand développement de l'ampoule rectale rappellent les stades larvaires correspondants décrits chez les Filarioidea.

Les papilles céphaliques sont déjà discernables. L'ensemble protorhabdion-osophage musculaire, difficile à séparer, mesure $30 \mu$, l'osophage glandulaire $65-70 \mu$. L'anneau nerveux parait être situé à $50 \mu$ de l'apex et le pore excréteur 5 à $10 \mu$ en dessous. L'ébauche génitale n'est pas visible; la queue, longue de $42-45 \mu$, est aiguë à pointe mousse. De très nombreuses granulations sont réparties dans tout le corps et gènent l'observation.

La larve parvenue à la fin du deuxième stade (Fig. 2 C) garde à peu près le même diamètre, bien qu'elle ait plus que doublé en longueur, atteignant une taille de 600 à $730 \mu$.

La cuticule commence à se décoller pour préparer la seconde mue ; elle déborde en avant (Fig. 2 D), mais, retenue par les bords du protorhabdion, elle se réfléchit pour former une cupule périorale. 
En arrière également, il existe un espace vide entre les deux cuticules, qui fait que la queue se termine par une petite vésicule réfringente.

Autour de l'anneau nerveux et du pore excréteur, apparaissent

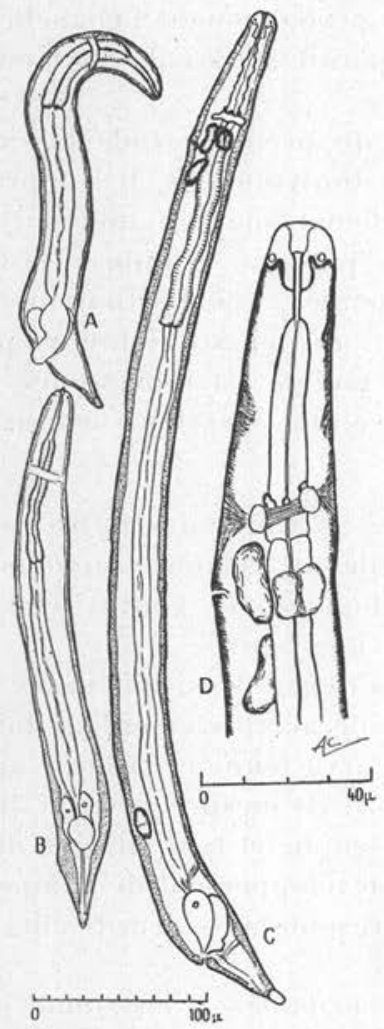

FIG. 2. - S. spinulatus. Deuxième stade larvaire. - A) Larve au $6^{\circ}$ jour ; vue latérale. - B) Larve au $6^{\circ}$ jour; vue ventrale. - C) Larve au $9^{e}$ jour; vue latérale. - D) Larve au $9^{\mathrm{e}}$ jour; extrémité antérieure, vue latérale.

de nombreux ganglions que nous désignerons suivant la terminologie employée par Alicata (1935) : céphaliques au-dessus de l'anneau nerveux ; latéraux et postéro-latéraux fusionnés; dorsaux peu apparents; les ventraux au contraire sont très développés, ils sont divisés par le pore excréteur en une masse préexcrétrice (subventraux et postéroventraux) et une masse postexerétrice (rétrovésiculaire).

L'ébauche génitale, formée de deux grandes cellules accolées, nous a paru être identique dans les deux sexes. Les diérides manquent.

Les mensurations suivantes sont prises sur une larve longue de 
$620 \mu$ : diamètre moyen, $33 \mu$; diamètre au niveau des glandes rectales, $40 \mu$; protorhabdion, $18 \mu$; œsophage musculaire, $50 \mu$; œsophage glandulaire, $120 \mu$; anneau nerveux à $68 \mu$ et pore excréteur à $95 \mu$ de l'apex ; ébauche génitale très postérieure, $85 \mu$ en avant de l'anus ; queue longue de $52 \mu$.

Troisième stade larvaire, - La larve du troisième stade est presque immobile lorsqu'elle est contenue à l'intérieur du Cypris, mais elle s'agite vigoureusement dès qu'elle est libérée dans l'eau. Elle reste vivante au moins 12 heures dans l'eau ordinaire.

La longueur totale varie de 1.250 à $1.750 \mu$. Les larves de petite taille ont été trouvées chez des Cypris multiparasités (7 à 12 larves); les plus grandes, au contraire, proviennent toujours d'hôtes peu parasités ( 1 ou 2 larves). La croissance de la troisième larve chez l'arthropode parait donc en réalité être extrêmement réduite.

Nous décrivons ci-dessous une larve longue de $1.380 \mu$ (Fig. $3 \mathrm{~A}$ ).

Corps longuement atténué en avant, le diamètre maximum de la partie postérieure étant de $45 \mu$. Cuticule épaisse à stries transversales fortes, espacées de $2,7 \mu$. Aires latérales bien visibles, larges de $13 \mu$.

L'extrémité antérieure prend la même organisation générale que chez l'adulte. Quatre papilles céphaliques submédianes petites; pseudo-lèvres saillantes à pointe obtuse ; protorhabdion très allongé $(80 \mu)$; anneau nerveux à sa place définitive, entourant le début de l'œsophage musculaire à $95 \mu$ de l'apex. Les ganglions nerveux ont la même disposition que chez la larve du second stade, mais les masses pré- et postexcrétrices ont fusionné. Une grosse cellule excrétrice est reliée par un fin canal long de $30 \mu$ au pore excréteur, qui s'ouvre à $110 \mu$ de l'extrémité antérieure. Les diérides situées $25 \mu$ plus en arrière ont la forme d'un aiguillon simple, à pointe dirigée postérieurement. Les cordons céphaliques paraissent être dejà indiqués par un épaississement cuticulaire au-dessus des papilles céphaliques. Wsophage musculaire long de $140 \mu$; œsophage glandulaire de $460 \mu$.

Les ébauches génitales peu organisées sont difficiles à étudier. Elles paraissent être représentées par un tube allongé, qui suit l'intestin depuis le rectum jusqu'au niveau de l'œsophage. Nous croyons cependant que la morphologie de l'extrémité postérieure peut suffire à différencier les sexes.

Dans un premier type (femelle ?), la région rectale est élargie (plus de $45 \mu$ ), la queue est épaisse, courte $(45 \mu)$ et tronquée (Fig. $3 \mathrm{C}$ ). 
Pour d'autres larves (màles ?), la région rectale est plus mince $(35 \mu)$, la queue est relativement longue $(50 \mu)$, digitiforme, à pointe arrondie (Fig. 3 B).

L'allongement des larves de grande taille porte surtout sur la région intestinale, et les mensurations concernant l'extrémité céphalique diffèrent peu des précédentes. On obtient, pour une larve de $1,75 \mathrm{~mm}$. : protcrhabdion, $85 \mu$; œsophage musculaire, $160 \mu$; resophage glandulaire, $450 \mu$; anneau nerveux, pore excréteur et diérides, respectivement à 100,120 et $135 \mu$ de l'apex.

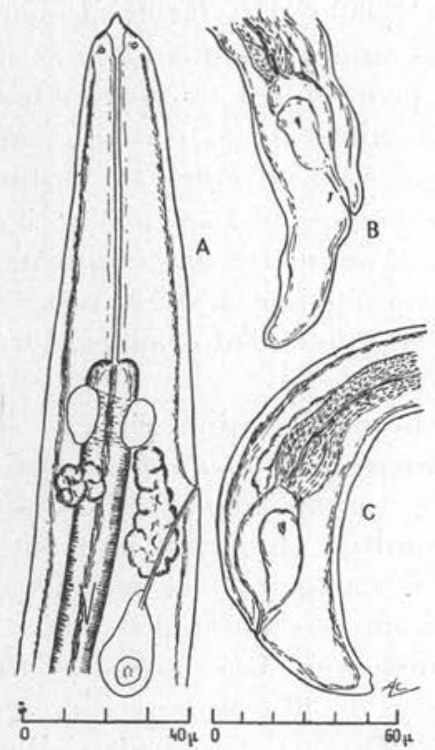

Fir. 3. - S. spinulatus. Troisième stade larvaire. - A) Larve au $11^{\circ}$ jour; extrémité antérieure, vue latẻrale. -

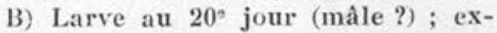
trémité postérieurc, vue latérale. C) Larve au $20^{\circ}$ jour (femelle ?) ; extrémité postérieure, vue latérale.

Quatrième stade larvaire, - Le matériel comporte 9 larves (3 mâles et 6 femelles), recueillies sous la membrane du gésier d'un garde-bœuf infesté depuis six jours.

Corps filiforme long de $2,33 \mathrm{~mm}$. (mâle) à $3,70 \mathrm{~mm}$. (femelle). La cuticule très épaisse présente des stries transversales fortes, espacées de 3,5 à $4,5 \mu$.

Extrémité céphalique (Fig. 4A) ornée de quatre cordons non récurrents, anastomosés deux par deux sur les faces latérales. Ces cordons sont grêles ; leur largeur, constante sur tout leur trajet, est d'environ $3 \mu$. Ils sont peu sinueux sur l'animal vivant ou fixé en extension. Chacun est formé par la juxtaposition de deux bandelettes ; la première, peu apparente, a une surface lisse ; la seconde, qui forme le bord interne de la courbe décrite par l'ensemble de deux 
cordons anastomosés, est striée finement dans le sens transversal, mais n'est pas spinulée.

Les deux paires de papilles submédianes sont très petites.

Les diérides extrêmement grandes $(45 \mu)$ sont tricuspides, la foliole centrale est deux fois plus longue que les folioles latérales (Fig. 4 B). Dans un cas, chez une larve màle, nous avons constaté une réduction marquée de la diéride droite ; longue de $20 \mu$ seulement, cette diéride n'est composée que de deux folioles atrophiées (Fig. $4 \mathrm{~A}$ ). Le còté gauche du même animal porte au contraire une diéride normalement développée.

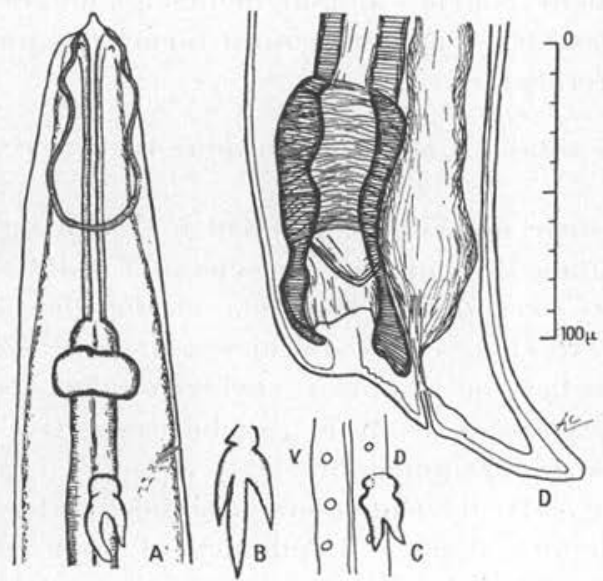

Fic. 4. - S. spinulatus. Quatrième stade larraire. - A) Larve mâle ingérée depuis 6 jours par Bubulcus ; extrèmité antérieure; vue latérale droite avec diéride atrophique. - B) Diéride gauche normale du même spécimen. C) Postdiẻride du même spéeimen. - D) Larve femelle ingérée depuis 6 jours par $\mathrm{Bu}$ bulcus ; extrémité postéricure, vue latérale.

Les postdiérides sont latéro-dorsales, insérées en arrière ou sur la partie dorsale de la ligne latérale (Fig. $4 \mathrm{C}$ ). La papille droite est située à peu près au niveau de la fin de l'œesophage ; la gauche est toujours largement plus postérieure. Ces postdiérides sont très remarquables par leur taille et leur forme. Elles sont bicuspides, ont une longueur de $20 \mu$ et sont donc très comparables à la diéride atrophique signalée plus haut. Cette similitude morphologique entre une papille cervicale et les papilles intestinales n'a pas à notre connaissance été signalée chez d'autres nématodes. Nous pensons qu'elle suffit à prouver l'analogie embryogénique des deux organes.

Le pore excréteur s'ouvre ventralement, un peu en avant des diérides. L'anneau nerveux entoure le début de l'œsophage musculaire.

Le tube digestif a la même constitution que chez l'adulte, avec un protorhabdion très allongé, un œesophage musculaire assez clair, un œsophage glandulaire atteignant environ la moitié de la longueur totale du corps; la portion terminale de l'osophage glanduiaire est moins foncée que la partie antérieure.

Les lignes latérales bien individualisées sont larges de $25 \mu$. 
Larve mâle. - Longueur totale, 2,33-2,62 $\mathrm{mm}$. Point de récurrence des cordons à $75-100 \mu$ de l'extrémité antérieure.

Protorhabdion long de 100-125 $\mu$. Portion musculaire de l'osophage Jongue de $280-315 \mu$ et portion glandulaire de $920-980 \mu$. Anneau nerveux et pore excréteur, respectivement à $120-150 \mu \mathrm{et}$ $150-180 \mu$ de l'apex. Diérides symétriques à $10-20 \mu$ en arrière du pore excréteur. Postdiéride droite située à peu près au niveau de la fin de l'oesophage $(110 \mu$ en avant ou $20 \mu$ en arrière). Postdiéride gauche postérieure de 320 à $360 \mu$ au point d'union osophagointestinal.

Queue cylindrique, légèrement courbée dorsalement, longue de 55-65 $\mu$. Phasmides non appréciables. A ppareil génital formé par un long tube cylindrique peu différencié.

Larve femelle. - Longueur totale, 3,7-4 $\mathrm{mm}$. Cordons récurrents à $100-110 \mu$ de l'apex.

Protorhabdion $130 \mu$. Esophage musculaire 320-350 $\mu$. Esophage glandulaire. $1,15-1,30 \mathrm{~mm}$. Milieu de l'anneau nerveux à $150-160 \mu$ de l'extrémité antérieure. Pore excréteur à $160-180 \mu$ et diérides à 180-200 $\mu$ de l'apex. Le pore excréteur et les diérides sont donc en général relativement plus proches de l'anneau nerveux chez la femelle que chez le mâle. Postdiérides droite et gauche, respectivement à 400 et à $975 \mu$ de la fin de l'œsophage.

L'extrémité postérieure (Fig. 4 D), brusquement atténuée, se termine par une queue longue de $50 \mu$, déjetée dorsalement. L'appareil génital est déjà entièrement constitué. La vulve occupe sa place définitive contre le bord antérieur de l'anus. Ovéjecteur légèrement dilaté, long de $220 \mu$, terminé par un sphincter puissant, long de $95 \mu$ et large de $45 \mu$. Trompe unique, longue de $280 \mu$, se continuant par une partie élargie, l'utérus, longue de $870 \mu$. L'oviducte, qui naît à $680 \mu$ en arrière de la fin de l'osophage, se prolonge par un ovaire rectiligne, remontant jusqu'au niveau de la portion glandulaire de l'œsophage. L’appareil génital femelle possède donc, dès son apparition, les particularités les plus remarquables de l'adulte : monodelphie et vulve en situation préanale.

\section{Conclusions}

1. - Morphogénèse des cordons céphaliques. - Seurat (1916 $a$, p. 348 et 367 ) a le premier montré qu'au cours du développement, les cordons céphaliques présentent des aspects correspondant à des formes plus primitives. En fait, il semble que la présence de ces formations soit un caractère assez rare chez les larves d'Acuaridæ. 
On n'en voit pas même sur le quatrième stade larvaire de formes très spécialisées, telle que Cheilospirura hamulosa (Diesing 1851) (cf. Alicata 1938, pl. 5, fig. F). Les seules larves munies de eordons céphaliques qui nous soient connues appartiennent à cinq̣ espèces :

a. Dispharynx spiralis (Molin 1858). Le troisième stade larvaire est dépourvu d'ornements. Mais Piana (1897) figure le quatrième stade larvaire préparant la dernière mue avec quatre cordons courts non récurrents, ni anastomosés, du type Acuaria.

b. Synhimantus (S.) laticeps (Rud. 1919). Le troisième stade larvaire est orné de quatre cordons comparables à ceux du quatrième stade de l'espèce précédente. Le dernier stade larvaire a la mème disposition que chez l'adulte (Seurat 1919). L'évolution est donc comparable à Dispharynx, mais la formation des cordons est réalisée plus précocement.

c. Synhimantus (S.) robertdollfusi Desportes 1947. Nous avons décrit (1950) une larve proche de la précédente, à diérides plus antérieures, trouvée encapsulée chez une couleuvre, et nous avons cru pouvoir la rattacher à cette espèce.

d. Acuaria tarentola Seurat $1916 \mathrm{~b}$. La larve placée sous ce nom a des cordons du même type que les précédents. Seurat avait suggéré un rapprochement avec Acuaria anthuris (Rud. 1819), mais le travail de Cram (1934) a prouvé qu'il n'en était rien. La place de cette larve est donc encore énigmatique et nous pensons qu'il faut la rapprocher des Synhimantus de rapaces.

e. Synhimantus (D.) spinulatus Chabaud et Campana 1949. Le troisième stade larvaire ne porte qu'une ébauche peu distincte en avant des papilles céphaliques. Le quatrième stade reste encore différent de l'adulte. Il porte des cordons anastomosés, mais non récurrents, comme on en voit chez Cheuvrexia.

L'évolution des cordons des Acuariidx, quand elle est observable, suit donc toujours un processus comparable passant des formes les plus primitives aux formes les plus évoluées. Le stade où apparaissent ces ornements est variable. Il est très précoce chez Synhimantus, plus tardif pour Desportesius et Dispharynx, mais le plus souvent ces formations n'apparaissent qu'au stade adulte.

2. - Développement et place zoologique. - Les deux faits qui nous paraissent les plus curieux dans ce cycle sont :

a. L'analogie morphologique du début du deuxième stade larvaire avec les stades en "saucisse 》 des Filarioidea. (Voir par exemple les figures données par Witenberg et Gerichter, 1944, pour une filaire d'amphibien Foleyella duboisi Gedoelst 1916). 
b. L'extrême rapidité du cycle. Nous avons à ce sujet publié une note (1949), en cherchant à prouver que les nématodes hétéroxènes les plus évolués ont une évolution plus rapide chez l'hôte intermédiaire et que, au contraire, les espèces hétéroxènes plus primitives n'atteignent le stade infectieux qu'après un séjour plus prolongé en dehors de l'hòte définitif. (Dans ce dernier cas, lorsque l'hòte intermédiaire a une existence éphémère (Copépodes, Ostracodes), le cycle habituel est modifié par différentes adaptations qui permettent une maturation complémentaire en dehors de l'arthropode vecteur).

Donc, aussi bien du point de vue morphologique que du point de vue biologique, l'étude des stades larvaires confirme les conclusions que, en collaboration avec Y. Campana, nous avions précédemment tirées de l'analyse morphologique de l'adulte: $S$. spinulatus est une espèce extrêmement spécialisée dans un sous-genre (Desportesius) lui-même très évolué. Nous ne devons donc pas nous étonner de trouver de nombreuses convergences avec les groupes de nématodes les plus évolués, tels que Echinurïnæ et mème Filarioidea.

3. - Réencapsulement. - En dehors de cctte rapidıté d'évolution, deux éléments contribuent à la conservation de l'espèce : d'une part, la longue conservation des œufs embryonnés dans l'eau ordinaire (au moins 7 jours); d'autre part, la faculté de réencapsulement.

Il est probable, étant donné le régime alimentaire des Ardéiformes, que, dans la nature, ceux-ci s'infestent plus fréquemment en ingérant des batraciens ou des poissons que par l'absorption de Cypris. Enfin, à l'inverse des Ostracodes, les vertébrés peuvent jouer, gràce à leur forte longévité, le ròle d'un "réservoir de virus » dans le cycle évolutif de cet helminthe.

Nous croyons pouvoir expliquer l'échec des expériences de réencapsulement chez les Gambusia par la rapidité du transit intestinal de ces poissons, plutôt que par une question de spécificité. On peut remarquer à ce sujet la rareté des helminthes adaptés aux Gambusia, aussi bien en France qu'au Maroc.

\section{REMERCIEMENTS}

Nous désirons exprimer toute notre reconnaissance au $\mathrm{D}^{r} \mathrm{G}$. Blanc, Directeur de l'Institut Pasteur de Casablanca, qui nous a fait le grand honneur de nous appeler en mission au Maroc. Nous remercions également très vivement le $\mathrm{D}^{r}$ Maurice pour notre séjour dans le Rharb, ainsi que MM. Assione, assistant à l'Institut Pasteur du 
Maroc, et Hugel, agent d'hygiène à Had Kourt, qui nous ont procuré les oiseaux utilisés pour ce travail.

\section{RÉSU MÉ}

Le nématode décrit précédemment sous le nom de Synhimantus (D.) equispiculatus Wu et Liu 1943, var. spinulatus Chabaud et Campana 1949 est élevé au rang d'espèce, car les spécimens récoltés au Maroc chez Bubulcus ibis s'éloignent de l'espèce equispiculatus, mais conservent de façon constante les caractères proposés pour la diagnose de la variété spinulatus.

Le cycle évolutif a été élucidé pour la première fois chez une espèce du genre Synhimantus. Les œufs embryonnés restent viables après un séjour d'une semaine dans l'eau. A l'exclusion d'autres Arthropodes, deux espèces d'Ostracodes ont permis l'évolution. Le iéencapsulement a été obtenu chez des têtards et un cyprinidé, mais non chez Gambusia sp. L'évolution, aussi bien chez l'hôte intermédiaire que chez l'hòte définitif, est extrêmement rapide et le cycle complet peut être fermé en moins de 28 jours. La morphologie des quatre stades larvaires a montré plusieurs caractères intéressants : épaississement du deuxième stade, diérides et postdiérides, cordons céphaliques.

L'étude morphologique et biologique des stades larvaires confirme les conclusions précédemment tirées de l'analyse morphologique de l'adulte : $S$. spinulatus est une espèce hautement spécialisée dans un sous-genre (Desportesius) qui est lui-même très évolué.

Nous trouvons donc de nombreuses convergences avec les groupes de nématodes les plus évolués, tels que Echinuriina et même Filarioidea.

\section{BIBLIOGRAPHIE}

Alicata (J. E.). - Early developmental stages of nematodes oceuring in sivine. U.S. Dept. Agr. Tech. Bull., 1935, n* 489, 96 p.

The life history of the gizzard worm (Cheilospirura hamulosa) and its mode of transmission to chickens, with special reference tc Hawaiian conditions. Livro Jub. Pr. L. Travassos, 1938, 11-19 + 5 pl.

Снaвaud (A.-G.). - A propos du cycle évolutif d'un Sỵnhimantus (NematodaAcuariida), recherche des lois qui régissent la vitesse du développement suivant la place zoologique du parasite et la biologie de i’hótc intermédiaire. Bull. Soc. \%oot, Fr., LXXIV, 1949, 342-345.

Sur le réencapsulcment 'des larves d'Acuariids. Vie et milieu, I, 1950. 
Chabaud (A.-G.) et Campana (Y.), - A propos d'une variété nouvelle de Synlimantus equispiculatus Wu et Liu 1943, création d'un nouveau sousgenre (Desportesius) n. subgen., Ann. Parasit., XXIV, 1949, 77-92.

Cram (E. B.). - Developmental stages of some nematodes of the Spiruroidea parasitic in poultry and game birds. U.S. Dept. Agr. Tech. Bull., 1931, $\mathrm{n}^{\circ} 227,27 \mathrm{p}$.

- Recent records of the gizzard worm, Acuaric anthutis (Rud. 1819) (Nematoda: Acuariidw), with observations on its life history. Proc. Helm. Soc. Wash., I, 1934, p. 48-49.

Cuvillerer (E.). - A new intermediate host for Cheilospirnra hamulosa, the gizzard worm of poultry. J. Parasit., XIX, 1933, 24\}-245.

Gexdne (E.). - Notes dhelminthologie africaine. (Quatrième note). Actes Soc. Linn. Bordeaux, LXVII, 1913, 106-112.

Hamaxs (O.). - Die Filarienseuche der Enten und der Zwischenwirt von Filaria uncinata R. Centralbl. Balt. Parasit., XIV, 1893, 555-557.

Puxa (G. P.). - Osservazioni sul Dispharagus nasutus Rud., dei polli e sulle larve nematoelmintiche delle mosche e dei porcellioni. Atti Soc. ital. sc. nat., XXXVI, 1897, 239-262.

Refuerzo (P. G.). - Arthropod intermediate host of Acuaria hamulosa in the Philippines, I. Nat. and Appl. Sc. Bull. Univ. Philippines, VII, 1940, 407-414.

Romavova (N. P.). - A study of the development cyele of Echinuria uncinata Rud. 18:9, a nematode parasitic of the stomach of Natatores. C.R. (Doli.) Acad. Sc. U.R.S.S, LV, 1947, 371-372.

Seurat (L.-G.). - Contribution à l'étude des formes larvaires des nématodes parasites hétéroxènes. Bull. Sc. Fr. et Belg., XLIX, 1916u, 297-377.

- Dispharages d'Algérie. C.R. Soc. Biol., LXXIX, 1916b, 934-938.

-- Contributions nouvelles à l'étude des formes larvaires des nématodes parasites hétéroxènes. Bull. biol. Fr. et Belg., LII, 1919, 344-378.

Witenzerg (G.) et Genichter (Ch.). - The morphology and life history of Foleyella duboisi with remarks on allied filarids of . Imphibia. J. Parasit., XXX, 1944, 245-256.

W'U (H. W. et Lie (C. K.). - Helminthological notes, III, Sinensia, XIV, 1943, 99-104.

Institut de Parasitologie de la Faculté de Médecine de Paris. (Directeur : Prof. H. Galliard) et Institut Pasteur du Maroc (Directeur: $D^{r}$ G. Blanc). 